\title{
Long-term Surgical Outcomes of Spinal Schwannomas: Retrospective Analysis of 49 Consecutive Cases
}

\author{
Erhan EMEL ${ }^{*}$, Anas ABDALLAH ${ }^{1 *}$, Ozden Erhan SOFUOGLU ${ }^{1}$, Ali Ender OFLUOGLU ${ }^{1}$, Muslum GUNES ${ }^{1}$, \\ Betul GULER ${ }^{1}$, Bilge BILGIC ${ }^{2}$ \\ ${ }^{1}$ Bakirkoy Research and Training Hospital for Neurology Neurosurgery and Psychiatry, Department of Neurosurgery, Istanbul, Turkey \\ ${ }^{2}$ Istanbul University, Faculty of Medicine, Department of Pathology, Istanbul, Turkey \\ *Drs. EMEL and ABDALLAH contributed equally to this work.
}

\section{ABSTRACT}

AIM: Spinal schwannomas (SS) represent the most common intradural extramedullary lesions, accounting for approximately $24 \%$ of all nerve sheath tumors in adults. Schwannomas have infrequent, but existent possibility of malignancy. In this study, long-term outcomes of 49 consecutive SS have been presented.

MATERIAL and METHODS: Medical records were retrospectively reviewed in 371 cases of spinal tumors who underwent surgery between the years 2005 and 2014. Cases confirmed as schwannoma histopathologically were included in this study. Patients' complaints, localizations, recurrence rate and complications were evaluated.

RESULTS: Forty-nine cases were detected in 47 (26 female, 21 male) patients. The mean age was $45.8 \pm 13.7$ years. The mean follow-up period was $61.4 \pm 21.5$ months. The most common complaint was local pain. Eleven were cervical, ten thoracic, twentyfour lumbar, and four in the sacral spine. Thirty-three cases were intradural-extramedullary, fifteen cases were the extradural type, and one case was the extra-intradural type. Recurrence rate was 4.08\%. Gross-total resection (GTR) was achieved in forty-seven patients. The most common complications were surgical site infection and intraoperative instability that were seen in three patients each. Posterior instrumentation was performed in two patients.

CONCLUSION: SS is mostly benign and intradural-extramedullary. To treat patients with SS, there is no need for adjuvant treatments; GTR with preservation of neurological functions is the best treatment to relieve patients' complaints and to reduce the recurrence rate of SS. To avoid serious complications, we recommend intraoperative neurophysiological monitoring and laminoplasty, especially in young patients. Dumbbell SS may require extensive bone resection. Posterior instrumentation can be used if instability occurs.

KEYWORDS: Spinal schwannoma, Intradural-extramedullary, Extradural, Gross-total resection, Laminoplasty

\section{INTRODUCTION}

S pinal schwannomas (SS) are well known, macroscopically solid encapsulated, spindle cell mesenchymal tumors which are called neurinomas in the literature. Although they are mostly benign entities derived from neoplastic schwann cells, SS have possibility of malignant transformation $(10,15)$. SS represent the most common intradural extramedullary lesions, accounting for about one fourth of all nerve sheath tumors, particularly in adults (8). Rarely, SS have possibility of malignancy and therefore the recommended primary treatment of choice is gross-total resection (GTR) with preservation of neurological function. GTR of these lesions, which is the main goal of surgical treatment, leads to good results.

Giant dumbbell SS, which extends anteriorly to invade the bones, vascular structures, and soft tissues and fill the abdominal and thoracic cavities as same as extradural location 
of SS rarely occurs (23). In 1952, Love and Dodge were the first authors to use the term 'dumbbell tumor' and report a neurogenic tumor with ingrowth into the spinal canal (18). This means that these tumors may grow extensively outside the spinal canal and compress muscles such as the paraspinal and iliopsoas as well as invade retroperitoneally (21).

SS that can occur in the spinal canal as well as those that develop in peripheral nervous system are almost solitary lesions (5). Microscopically, schwannomas are characterized by high cellularity, and relative lack of an Antoni A and Antoni $B$ areas. SS are usually strong positive for the $S-100$ protein (10). The multiple forms of neurofibromas is known as von Recklinghausen's disease (3). Neurofibromatoses are genetic disorders that affect nerve cell tissues (9). The incidence of SS varies between 3-4 cases/1,000,000 persons per year (22). SS typically display no gender preponderance while the mean of age of appearance is usually between $40-50$ years with the exception of a few cases $(2,4,5,22)$.

GTR of SS allows good clinical outcomes in patients in which the preoperative clinical findings are not particularly severe. Herein, the results of surgical treatment and longterm outcomes of our 49 consecutive SS cases have been evaluated.

\section{MATERIAL and METHODS}

This retrospective, randomized study was approved by the medical ethics committee of our hospital. Written informed consent was obtained from all patients for publication of their records and images.

Medical records were retrospectively reviewed in 371 cases of spinal tumors who were operated in our hospital at the department of neurosurgery, between the years 2005 and 2014, and the cases confirmed as schwannoma histopathologically were included in this study. The long-term clinical outcomes were evaluated retrospectively using patients' complaints, tumor locations, recurrence rate and complications. All patients underwent a hemilaminectomy, laminectomy or laminotomy before tumor removal. To avoid serious complications, intraoperative neurophysiological monitoring and laminoplasty were performed, especially in the last four years.

Data are expressed as mean \pm standard deviation values of the mean, median, and minimum-maximum. Differences among groups were assessed with one-way analysis of variance (ANOVA) using the SPSS 21.0 statistical package. Differences were considered statistically significant when $p$ was $<0.05$.

The clinical, radiological and surgical features of 3 patients are presented as illustrative cases for SS.

\section{Illustrative Cases}

Case 1: A 25-year-old female patient presented with low back and bilateral leg pain. Magnetic resonance imaging (MRI) with contrast demonstrated a well-circumscribed, large sacral extradural mass measuring $97 \times 88 \times 81 \mathrm{~mm}$, associated with neural tissues and showing heterogeneous enhancement after contrast on both T1- and T2-weighted images. The mass extended to the pelvic space (Figure 1A). The patient underwent 2-step surgery; in the first step, the extradural mass lesion was resected posteriorly and in the second step the remaining anterior part of tumor was removed using an anterior approach. Total resection was therefore performed. Histopathologically, the mass lesion was confirmed to be a schwannoma. The patient was well and neurologically intact. She was discharged to home on postoperative Day 4. Postoperative imaging demonstrated complete resection of the mass and no evidence of a pseudomeningocele (Figure 1B). The patient had complete resolution of her symptoms. After 7 months, she presented with back pain. MRI demonstrated a suspect recurrent lesion or fibrosis tissue (Figures 1C, D). The patient underwent a third operation and the histological diagnosis was fibrosis.

Case 2: A 70-year-old woman presented with back and right leg pain. MRI showed a well-circumscribed mass measuring $27 \times 28 \times 16 \mathrm{~mm}$, which eroded the right pedicle of the L4 vertebra and narrowed the right $L 4$ foramen. The mass showed heterogeneous enhancement after contrast on both T1- and T2-weighted images (Figure 2A). She underwent right L4 laminectomy and it was intraoperatively observed that the lesion was intradural extramedullary. GTR was achieved. The estimated blood loss was $300 \mathrm{ml}$. The patient was neurologically intact and discharged on the postoperative second day. Postoperative MRI did not show any evidence of recurrence or pseudomeningocele (Figure $2 \mathrm{~B}$ ).

Case 3: A 36-year-old woman presented with back and chest pains for more than 6 months. A suspected, well-circumscribed mass was seen in performed X-rays (Figure 3A). MRI showed a well-circumscribed firm mass located on the lamina of T10 (Figure 3B). The patient subsequently underwent right $10^{\text {th }}$ thoracic laminotomy, and GTR of the brownish solid soft encapsulated mass (Figure $3 \mathrm{C}$ ) was achieved. The histological diagnosis was schwannoma (Figure 3D-F).

\section{RESULTS}

Forty nine spinal schwannoma cases were detected in 47 patients, 26 female (57.1\%) and 21 (42.9\%) male (Figure 4). The mean age was $45.8 \pm 13.7$ years (range 24 and 82 years) (Figure 5). The mean follow-up period was 61.4 \pm 21.5 (9-108) months (Table I). The most common complaints were local pain $(85.7 \%)$, radicular pain, myelopathy, loss of sensation, motor deficit, incontinence and myoclonus (Table II). Eleven [Intradural-extramedullary (I): 7, Extradural (E): 4] [22.5\%] cases were sited in the cervical spine, ten (I:6, E:4) [20.4\%] were thoracic, 24 (I:20, E:4) [49\%] were lumbar, and 4 (I:0, E:4) [8.1\%] were in the sacral spine (Table III). Thirty-three (67.4\%) cases were the intradural-extramedullary type (while one of them was extended to thoracic space, three cases extended to the pelvic space; they ought to be called giant dumbbell SS), fifteen (30.6\%) cases were the extradural type, and one case was the extra-intradural type (2\%) (Table III). Two cases (4\%) showed recurrence in the same patient who underwent 3 operations. 
Table I: Baseline Clinical and Demographic Characteristics of the 47 Patients

\begin{tabular}{|c|c|c|c|}
\hline & Female & Male & All pts \\
\hline No. of Patients & 26 pt $(57.1 \%)$ & $21 \mathrm{pt}(42.9 \%)$ & 47 pt $(100 \%)$ \\
\hline Age (years) & $\begin{array}{c}45.43 \pm 13.89 \\
\text { (range: } 24-70)\end{array}$ & $\begin{array}{c}46.63 \pm 14.79 \\
\text { (range: } 25-82 \text { ) }\end{array}$ & $\begin{array}{c}45.82 \pm 13.67 \\
\text { (range: } 24-82 \text { ) }\end{array}$ \\
\hline Symptom duration & $\begin{array}{c}1 \text { day }-3 \text { years } \\
\text { (Av. } 11.26 \pm 10.02 \text { months) }\end{array}$ & $\begin{array}{c}2-60 \text { months } \\
\text { (Av. } 16.03 \pm 17.33 \text { months) }\end{array}$ & $\begin{array}{c}1 \text { day }-5 \text { years } \\
\text { (Av. } 13.24 \pm 13.54 \text { months) }\end{array}$ \\
\hline Follow-up period (months) & $\begin{array}{c}\text { 9-108 month } \\
\text { (Av. 61.06 } \pm 20.86)\end{array}$ & $\begin{array}{c}\text { 11-106 month } \\
\text { (Av. } 62.94 \pm 22.65)\end{array}$ & $\begin{array}{c}\text { 9-108 month } \\
\text { (Av. 61.38 } \pm 21.52)\end{array}$ \\
\hline Mean length of hospital stay (day) & $4.18(2-15)$ & $9.4(2-31)$ & $6.4(2-31)$ \\
\hline Mean intraoperative blood loss (cc) & $544(280-2650)$ & $1048(50-4500)$ & $760(50-4500)$ \\
\hline
\end{tabular}
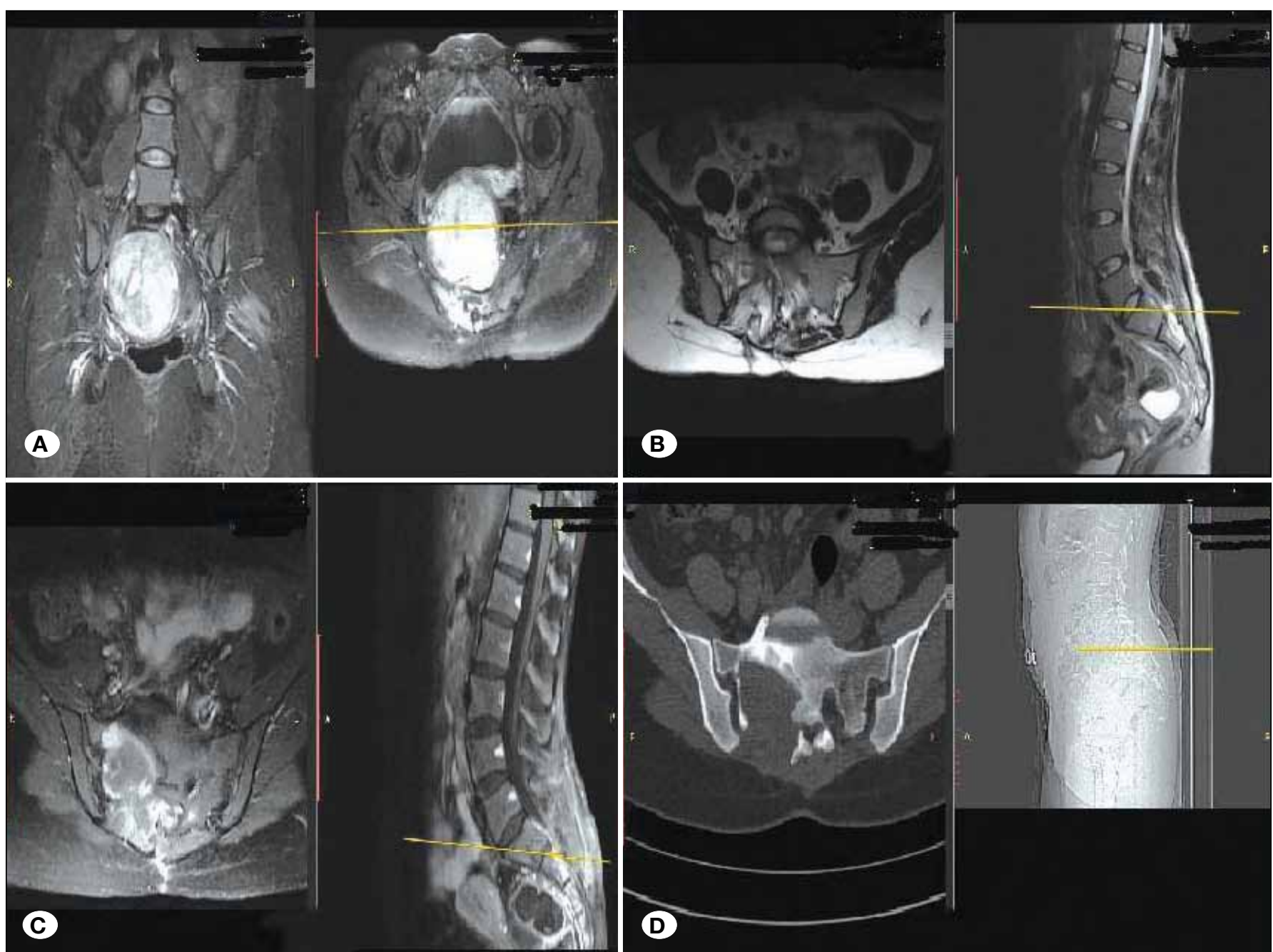

Figure 1: A 25-year-old female patient presented with low back and bilateral leg pain, hemisensory changes and gait difficulty. A) MRI with contrast demonstrated a well-circumscribed, large sacral extradural mass measuring $97 \times 88 \times 81 \mathrm{~mm}$, associated with neural tissues and showing heterogeneous enhancement after contrast on both T1- and T2-weighted images. Note that the mass extends to the pelvic space; B) Postoperative MRI demonstrates complete resection of the mass and no evidence of a pseudomeningocele; C) Postoperative seventh-month MRI demonstrates a recurrent lesion that was thought to be recurrent schwannoma and later was histopathologically confirmed to be fibrosis; D) Postoperative CT after the second surgery shows that the erosions happened secondary to SS. 


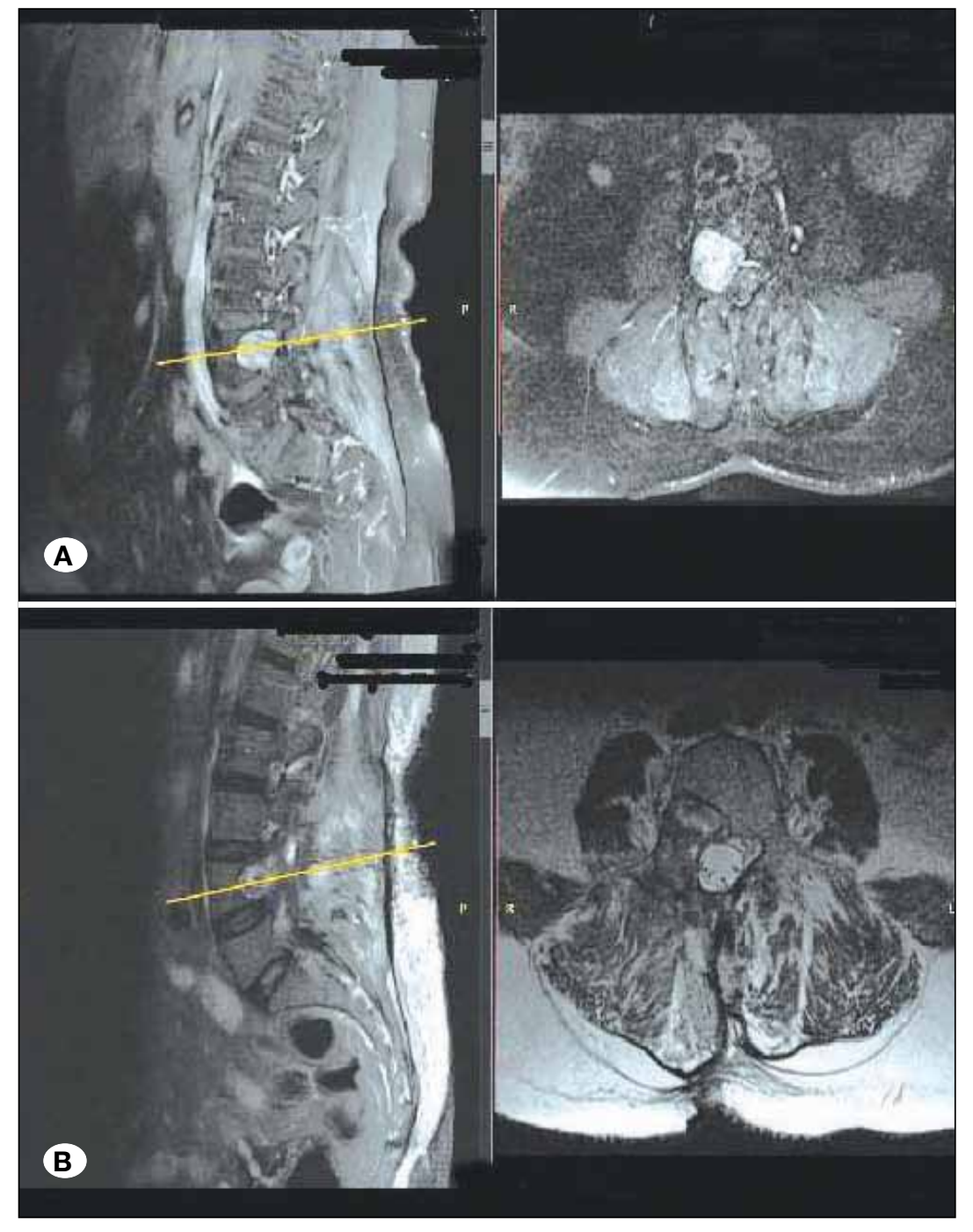

Figure 2: A 70-year-old female patient presented with back and right leg pain. A) MRI showed a well-circumscribed mass measuring $27 \times 28 \times 16 \mathrm{~mm}$ that eroded the right pedicle of the L4 vertebra and narrowed the right $L 4$ foramen;

B) Postoperative MRI did not show evidence of recurrence or pseudomeningocele.

Table II: Complaints of the 49 Cases

\begin{tabular}{lccc}
\hline Complaint & Female (n=28) & Male (n=21) & All pts (n=49) \\
\hline Local pain & $82.1 \%(23)$ & $90.5 \%(19)$ & $85.7 \%(42)$ \\
\hline Radicular (extremity) pain & $75 \%(21)$ & $61.9 \%(13)$ & $69.4 \%(34)$ \\
\hline Myelopathy & $35.7 \%(10)$ & $33.3 \%(7)$ & $34.7 \%(17)$ \\
\hline Loss of sensation & $28.6 \%(8)$ & $33.3 \%(7)$ & $30.6 \%(15)$ \\
\hline Motor deficit & $32.1 \%(9)$ & $23.8 \%(5)$ & $28.6 \%(14)$ \\
\hline Urinary incontinence & $10.7 \%(3)$ & $9.5 \%(2)$ & $10.2 \%(5)$ \\
\hline Myclonus & $10.7 \%(3)$ & $9.5 \%(2)$ & $10.2 \%(5)$ \\
\hline Neurogenic Claudication & $7.1 \%(2)$ & $9.5 \%(2)$ & $8.2 \%(4)$ \\
\hline Gait impairment & $7.1 \%(2)$ & $4.8 \%(1)$ & $6.1 \%(3)$ \\
\hline Headache & 0 & $9.5 \%(2)$ & $4.08 \%(2)$ \\
\hline Chest pain & 0 & $4.8 \%(1)$ & $2.04 \%(1)$ \\
\hline Swelling in neck & 0 & $4.8 \%(1)$ & $2.04 \%(1)$ \\
\hline
\end{tabular}




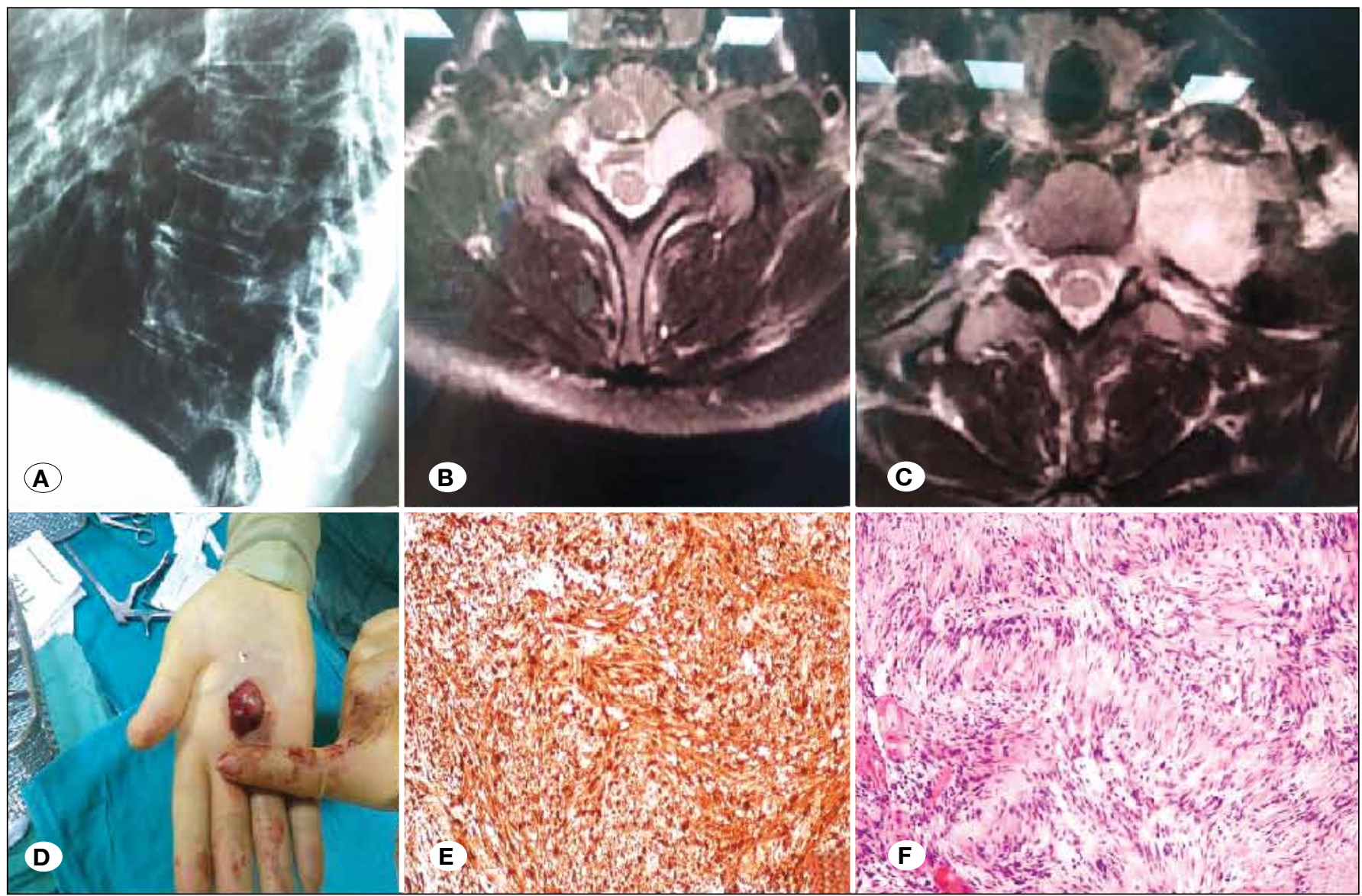

Figure 3: A 36-year-old female patient presented with back and chest pain. A) A well-circumscribed mass was seen in the X-rays; B,C) MRI showed a well-circumscribed firm mass located on the lamina of T10; D) Macroscopic view of the mass (brownish solid soft encapsulated mass); E) Histopathological examinations: diffuse immunohistochemical S-100 positivity was shown (x100); F) Histopathological examinations: Hematoxylin-eosin stained sections revealed a spindle cell tumor (x200). S-100 positivity confirmed the diagnosis of Schwannoma.

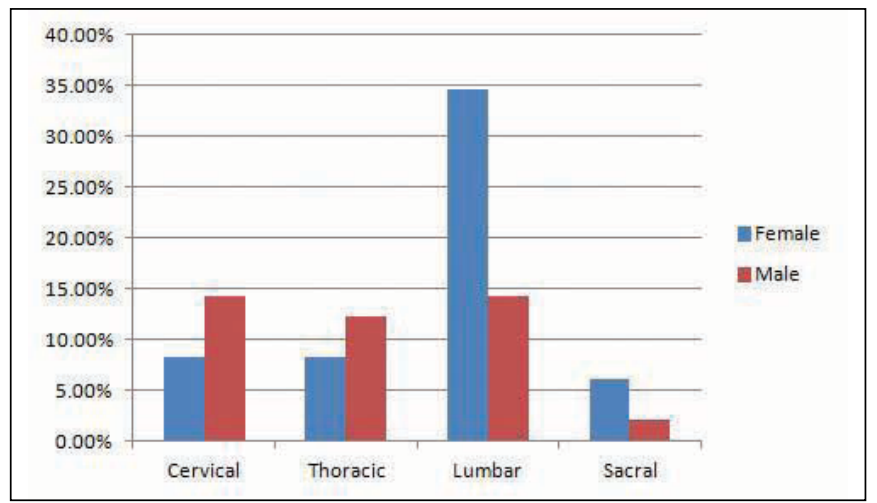

Figure 4: Locations of spinal schwannomas.

The mean time interval between the first complaint and surgery was 12.9 months (1 day-60 months). Co-morbidity factors and the body mass index (BMI) of the 47 patients are listed in Table IV. The mean length of stay in hospital was 6.4 (2-31) days. Forty seven patients underwent 59 operations; 47 as primary, 5 as secondary to achieve gross-total resection, 2 for recurrences, and 2 for abdominal abscess and repair of

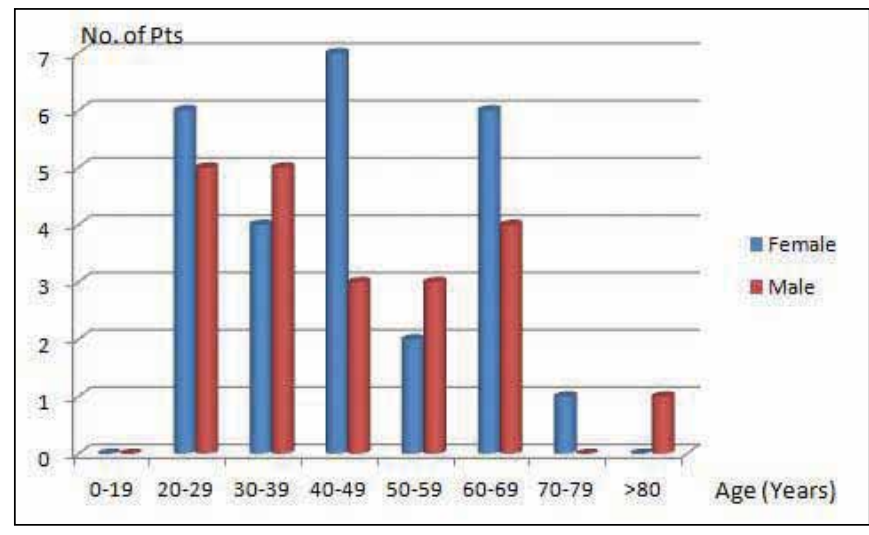

Figure 5: Distribution of the patients regarding to age group and gender of the patients.

surgical site. There were also three more operations which were performed in three giant dumbbell SS that were thought to be recurrences. However, the histological diagnosis of the materials obtained intraoperatively was fibrosis, and there was no evidence of recurrent lesions. The majority of our 
approaches were posterior (54/59) at $91.5 \%$. The anterior approach was only used for cervical spinal SS and to remove tumors that extended to both thoracic and pelvic spaces. Intraoperative instability was seen in 3 patients while posterior instrumentation was performed in 2 patients. One patient was still without posterior fusion because the pedicles were eroded by the tumor and a back brace had been recommended after surgery. Five cases complained that the neurological deficits (28/49) had not improved completely (Table V). GTR was achieved in forty-three patients, subtotal resection (STR) in five patients, and one patient underwent surgery for biopsy and then he refused a second operation. The patient who had been managed as STR underwent a second surgery and 4 out of 5 cases had been resected totally. Surgical site infection was seen in three patients. One of them presented with abdominal abscess while another patient presented with meningitis and they got treated successfully. The mean loss of blood was $760 \mathrm{cc}$.

Table III: Localizations of the 49 Cases

\begin{tabular}{cccc}
\hline & Female & Male & All pts \\
\hline Spinal location • Cervical: & $4(8.2 \%)$ & $7(14.25 \%)$ & $11(22.45 \%)$ \\
\hline$\bullet$ Thoracic: & $4(8.2 \%)$ & $6(12.2 \%)$ & $10(20.4 \%)$ \\
\hline - Lumbar: & $17(34.7 \%)$ & $7(14.3 \%)$ & $24(49 \%)$ \\
\hline - Sacral: & $3(6.11 \%)$ & $1(2.04 \%)$ & $4(8.15 \%)$ \\
\hline Location according dura & & & $33(67.35 \%)$ \\
\hline$\bullet$ Intradural-extramedullary: & $19(38.78 \%)$ & $14(28.57 \%)$ & $15(30.61 \%)$ \\
\hline & $9(18.37 \%)$ & $6(12.24 \%)$ & $1(2.04 \%)$ \\
\hline
\end{tabular}

Table IV: Co-Morbidities and BMI of the 47 Patients

\begin{tabular}{|c|c|c|c|}
\hline & Female (26) & Male (21) & All pts (47) \\
\hline $\mathrm{BMI}\left(\mathrm{kg} / \mathrm{m}^{2}\right)$ & $\begin{array}{c}25.68 \pm 3.41 \\
\text { (range: } 17.2-30.1 \text { ) }\end{array}$ & $\begin{array}{c}27.88 \pm 1.76 \\
\text { (range: } 22.1-28.1 \text { ) }\end{array}$ & $\begin{array}{c}25.63 \pm 2.46 \\
\text { (range: } 17.2-30.1 \text { ) }\end{array}$ \\
\hline Smoking & 7 pts $(26.9 \%)$ & 13 pts $(61.9 \%)$ & 20 pts $(42.6 \%)$ \\
\hline Hypertension & 10 pts $(38.5 \%)$ & 4 pts (19\%) & 14 pts $(29.8 \%)$ \\
\hline Thyroid dysfunction & 4 pts $(15.4 \%)$ & 2 pts $(9.5 \%)$ & 6 pts $(12.8 \%)$ \\
\hline Diabetes Mellitus & 4 pts (15.4\%) & 1 pt (4.8\%) & 5 pts (10.6\%) \\
\hline Coronary artery diseases & 2 pts $(7.7 \%)$ & 0 & 2 pts $(4.3 \%)$ \\
\hline Multiple sclerosis & 1 pt $(3.8 \%)$ & 0 & 1 pt $(2.1 \%)$ \\
\hline Past history of Stroke & 1 pt $(3.8 \%)$ & 0 & 1 pt $(2.1 \%)$ \\
\hline Prior Surgery & 7 pts (26.9\%) & 13 pts $(61.9 \%)$ & 20 pts $(42.6 \%)$ \\
\hline
\end{tabular}

Pt: Patient, BMI: Body mass index.

Table V: Surgical Treatment Outcomes

Improvement (Surgical treatment outcome)

Number of Cases (\%)

Total resection with good recovery $36(73.48 \%)$

Total resection with improvement in clinical symptoms

$4(8.16 \%)$

Total resection with worsened clinic

$1(2.04 \%)$

Residue then total resection after $2^{\text {nd }}$ surgery with good recovery

$4(8.16 \%)$

Residue after $2^{\text {nd }}$ surgery without recurrence

$1(2.04 \%)$

Biopsy then patient refused $2^{\text {nd }}$ operation

$1(2.04 \%)$

Despite of total resection recurrent cases (treated with good recovery)

$2(4.08 \%)$ 


\section{DISCUSSION}

Most schwannomas (neurinomas) are solid and firm neoplasms that originate from neoplastic Schwann cells (10). These softtissue lesions are usually benign, slow-growing and do not grow greater than $8 \mathrm{~cm}$ in diameter (6). SS are asymptomatic for a long time due to the slow tumor growth rate, and rarely giant SS, which are most often discovered in the cauda equina but can also occur at other sites, develop because of the chance of the tumor to grow insidiously to a large size without causing any compression of the nerve roots (12).

Neurofibromatosis (NF) is a genetic disease that affects the development and growth of nerve tissues that may then develop heterogeneous tumors. There are three types of NF; NF-1, NF-2, and NF-3 (schwannomatosis) which is characterized by multiple cutaneous and spinal schwannomas without acoustic tumors or other signs of NF-1 or NF-2 (9). None of our patients was diagnosed as NF-1, NF-2 or as schwannomatosis (NF-3).

In our series of spinal canal tumors that underwent surgery consecutively between 2005 and 2014, SSs account for about $13.2 \%$ (49/371), while they account for about $31.8 \%$ (34/107) of primary intradural spinal cord tumors. In a review of the literature this percentage ranged between $25 \%$ and $33 \%$ $(3,5,8,10,15,21,22)$. The literature review showed that there is no significant prevalence difference between males and females $(2-5,22)$. However, the authors noticed a somewhat higher prevalence in females, accounting for 26 patients (57.1\%). The incidence of SS varies with the age of affected patients who are between the $4^{\text {th }}$ and the $5^{\text {th }}$ decade $(10,22)$. In our series, the mean age was $45.8 \pm 13.7$ years (ranged 24-82 years), so SS affects a large age range (Figure 5). Schwannomas may present in all parts of the spine (22), even if the major locations have been reported as the cervical and lumbar regions $(5,10,22)$. In our study, the highest incidence was in the lumbar region (49\%), followed by the cervical $(22.5 \%)$ and thoracic spine (20.4\%). In the literature, $70 \%$ to $80 \%$ of SSs are reported to be intradural in location, and those extending through the dural aperture as a dumbbell-shaped mass with both intradural and extradural components account for another $15 \% \quad(11,20)$. Intramedullary schwannomas are extremely rare (2). The authors were not able to find intramedullary schwannoma although 33 (67.4\%) cases were of the intradural-extramedullary type (while one of them was extended to the thoracic cavity, three cases else extended to the pelvic cavity; they ought to called giant dumbbell SS); 15 $(30.6 \%)$ cases were the extradural type, and one case was the extra-intradural type (2\%).

The initial symptoms are varied in accordance with the compressed nerve roots of the tumor as well as varied the roots it originated from. The most common complaints among our patients were local pain $(85.7 \%)$, radicular pain (69.4\%), myelopathy, loss of sensation, motor deficit and incontinence, respectively (Table I). The pain was mostly on the tumor site, sometimes spread to both sides, mostly temporary, but always in the same place. At the beginning, the local pain is due to the disturbance of nerve conductivity because of the direct or indirect irritation of the nerve root or root compression by the mass of lesion $(2,21)$. With time, the enlarged mass will lead to compression of the spinal cord even if the tumor grows slowly and as a result, the spinal tracts get damaged and myelopathy develops (10). However, motor deficits rarely occur as an initial symptom, particularly in the tumors of lumbosacral region. Motor weakness of the lower extremities may not be obvious until the later stage, as in patients with lumbar canal or foraminal stenosis. Although GTR of spinal nerve sheath tumors has been considered to be feasible $(7)$, some cases have resected subtotally $(3,17)$. There may be many obstacles to GTR such as adhesion to the spinal cord because of hemorrhage, inflammation, or subpial localization (10); the other obstacle is critical structures attached to extradural components outside the spinal canal, such as the vertebral artery in cervical tumors and abdominal aorta in lumbar tumors. Giant sized tumors and invasion of the bone and facet joints may be other obstacles in surgery. In our series, postoperative first day enhanced-MRI with contrast was performed to determine if GTR was achieved or not. GTR was not achieved in six cases and 5 of them underwent second surgery for GTR, while GTR was achieved in fortythree cases at the first surgery. Thus GTR was achieved in 47 out of 49 cases (95.9\%). One patient underwent surgery for biopsy and then he refused the second operation. The other patient underwent two operations and GTR could not achieved because of vascular structures. The patient has been called back yearly and MRI performed and his tumor has not recurred by now (postoperative sixth year). In such patients, long-term observation is needed (11). However, study MRI well as same as a good knowledge of the anatomy of surrounding structures and selecting the appropriate surgical techniques and approach could overcome these obstacles. The high rate of GTR between our patients was related to the use of intraoperative neurophysiological monitoring (IONM) that let surgeons act more safely. Only one case operated without using IONM got worsen neurological clinic even the patient was presented with myelopathy and motor deficits.

Postlaminectomy kyphosis is highly correlated with removal of facet joints bilaterally, after laminectomy, by time the degree of angulations gets increased (16). In two cases of our cases even no facets were removed, a gradual intraoperative stability was developed which had been treated with posterior instrumentation. Although recommended laminectomy to be less than $12 \mathrm{~mm}$, bilateral facetectomy at one or more levels, may lead to make acute angular kyphosis. Despite the high control of tumor recurrence, unilateral facetectomy may predispose to a deformity in the spine such as angular kyphosis or scoliosis with angular kyphosis which may both lead to spinal cord compression (16). In the review of the literature, many surgeons recommended laminoplasty. However, there is not enough data to confirm the effectiveness of the procedure. For example; Abbott et al. reported the results of laminoplasty for spinal cord tumors in a few children (1). Another study by Kim et al. showed that priority of the laminoplasty to prevent postoperative spinal deformities after removal of spinal cord tumors in 16 cases compared with 89 cases of laminectomy (14). The Kawahara et al. study is another evidence of laminoplasty priority. They performed recapping T-Saw laminoplasty for spinal cord tumors in 24 patients, 
and they reported no complications such as postoperative spinal canal stenosis, facet arthrosis, or kyphosis (13). Jeon et al. (10) performed laminoplasty on C2-C3 and L2-L3, in two separate cases, and they reported that it could be helpful to dissect the epidural space at the time of second operation due to recurrent lesions on L4. In adults, removal of the facet joints unilaterally for tumors located in the cervical or thoracic spine does not require fusion, but might be necessary for those located in the lumbar spine (10). Particularly, in the last 4 years, the authors ought to use laminoplasty rather than laminectomy. Laminotomy using high speed drills to reach the tumor and resect it, followed by sutured lamina separated layers using a strong non-absorbable suture and laminoplasty was performed in 19 patients in whom none experienced spinal deformities up to now [The mean follow-up period was $28.8 \pm 15$ months (9-51 months)], with no significant difference $(p=0.08)$, while in 3 out of the remaining 30 cases intraoperative instability was seen. While posterior instrumentation was performed in 2 patients, one patient was still without posterior fusion because the pedicles were eroded by the tumor and back bracing had been recommended after surgery.

Several approaches have been described for resection of SS such as mini-open (minimal invasive), traditional open laminectomy or laminoplasty, A wide laminectomy is still the standard approach used to explore extra- and intradural compartments of spinal tumors. In a comparison between laminoplasty (laminae were bilaterally removed using high speed drill, then tumor resection was performed, and laminae were bilaterally sutured using a strong non-absorbable suture to both laminae parts and interspinous ligaments cranially and caudally), hemilaminectomy and laminectomy in our series, we found that laminoplasty was better than hemilaminectomy and laminectomy approaches in the length of stay at hospital with averages of $2.9 \pm 1.05(2-5)$ days, against $5 \pm 2.16$ (28) days, and $9.5 \pm 7.17$ (3-31) days, respectively (Table VI). This difference was not statistically significant $(p=0.33$ and $p=0.4$, respectively) (significance level was considered as $\mathrm{p}<0.05)$. However, the mean intraoperative blood loss was less in laminoplasty with an average of $395 \mathrm{cc}$ and in hemilaminectomy with average of $435 \mathrm{cc}$ versus an average of $1130 \mathrm{cc}$ in cases who underwent total laminectomy (Table VI). These values were significantly different $(p<0.001)$. Actually, the five second surgeries to achieve GTR in total laminectomy group lead to increased mean intraoperative blood loss per case. On the other hand, the three approaches did not affect the neurological outcomes and surgery-related complications. One patient of each group experienced surgical site infection. Intraoperative instability was seen in two patients who underwent total laminectomy and another one who underwent hemilaminectomy.

Forty seven patients underwent 59 separate operations; 47 as primary, 5 as secondary to achieve GTR, 2 for recurrences, and 2 for abdominal abscess and repair of the surgical site. There are three operations else were perfomed in three giant dumbbell SS that thought to be recurrences (patients experienced the same complaints that they were experiencing before the first surgery. The new MRI showed a mass that was suspected to be a recurrence. No enhanced MRI was performed. Patients were reoperated to relieve the complaints), but perioperatively the materials obtained were confirmed as fibrosis histopathologically, and there was no evidence of recurrent lesions. The majority of our approaches were posterior $(54 / 59)$ at $91.5 \%$. The anterior approach was only used for cervical spinal SS and to remove tumors that extended to both the thoracic and pelvic cavities.

Recently, intraoperative neurophysiological monitoring (IONM) in spinal surgery has become more popular. Twenty-five cases (15 females and 10 males) out of 49 cases were treated using IONM. Total resection was confirmed by enhanced MRI in all cases. Twenty-three cases recovered well and 2 cases were improved. In contrast, one patient out of 24 patients who were treated without using IONM got worse clinically and was therefore referred to rehabilitation program (Table

Table VI: Comparison Between Surgical Approaches Used to Treat the Patients in This Series

\begin{tabular}{lccc|ccccc|c|c}
\hline Approach & \multicolumn{3}{c}{ No. of patients } & \multicolumn{3}{c}{ Localization } & Hospital stay & Blood loss \\
\hline & Female & Male & All pts & Cervical & Thoracic & Lumbar & Sacral & (days) & (cc) \\
\hline Laminoplasty & 9 & 10 & 19 & 4 & 5 & 9 & 1 & 2.9 & 395 \\
\hline Hemilaminectomy & 5 & 2 & 7 & 2 & 1 & 3 & 1 & 5 & 435 \\
\hline Total laminectomy & 14 & 9 & 23 & 5 & 4 & 12 & 2 & 9.5 & 1130 \\
\hline
\end{tabular}

Table VII: Surgical Outcomes Using Intraoperative Neurophysiological Monitoring (IONM)

\begin{tabular}{ccc|cccc|cc|ccc}
\hline Using & \multicolumn{2}{c|}{ No. of patients } & \multicolumn{4}{c|}{ Localization } & \multicolumn{2}{c}{$\mathbf{1}^{\text {st }}$ Surgery } & \multicolumn{3}{c}{ Surgical treatment outcomes } \\
\hline IONM & Female & Male & Cervical & Thoracic Lumbar & Sacral & GTR & STR & Recover & Improve Unchange & Worsen \\
\hline Yes & 15 & 10 & 5 & 6 & 12 & 2 & 25 & - & 23 & 2 & - \\
\hline No & 13 & 11 & 6 & 4 & 12 & 2 & 18 & $6^{*}$ & 13 & 2 & $1^{* *}$ \\
\hline
\end{tabular}

*STR: Subtotal resection; STR was performed in five patients, and one patient underwent surgery to get biopsy then he refused second operation.

${ }^{* *}$ The patient who underwent biopsy. 
VII). Five cases of those complaining of neurological deficits (28/49) had not improved completely, whereas the symptoms and signs of our patients improved in 95.9\% (47/49) (Table V). GTR was achieved in forty-three patients, subtotal resection (STR) in five patients, and one patient underwent surgery for biopsy and then refused the second operation. The patients who had been managed as STR underwent a second surgery and 4 out of 5 cases had been resected totally. Using IONM may facilitate gross-total resection $(p=0.0016)$ (Table VII). The surgical treatment outcome of SS correlates with the preoperative neurological condition of the patient (19). GTR of SS is almost curative unless SSs are associated with neurofibromatosis. However, dumbbell-shaped tumors with extensive muscle (e.g. paraspinal) involvement that are subtotally resected have a definite high risk of recurrence. Some studies have shown that the deficits resulting from sacrifice of the involved nerve roots are usually not permanent and well tolerated $(7,22)$. Although recurrence is usually seen in cases where STR was achieved, the female patient who had recurrent SS after 36 and 55 months was treated by GTR twice. Otherwise, the patient treated with STR after two surgeries did not experience recurrence. Surgical site infection was seen in three patients. One of them presented with abdominal abscess while another patient presented with meningitis and they got treated successfully. The mean blood loss was $760 \mathrm{cc}$ (Table I).

Although the high incidence of postoperative neurological deficits can be expected in the dumbbell-shaped SS patients (21), our three patients did not experience such complications. The authors related this to resecting these lesions in two separate surgeries; the first one via a posterior, and the second via an anterior approach. Dumbbell-shaped SS patients should be carefully treated in two operations if necessary.

\section{CONCLUSION}

Spinal schwannoma is mostly benign, slow-growing and intradural-extramedullary tumor. Schwannomas accounted for $13.2 \%$ of our series of spinal tumors. GTR with preservation of neurological functions is the best way to relieve patients' complaints and to reduce the recurrence rate of SS. To avoid serious complications, the authors recommend intraoperative neurophysiological monitoring and laminoplasty, especially in young patients. Schwannomas have infrequent, but existent possibility of malignant transformation. Therefore, GTR is the treatment of choice for patients with SS. Postoperative MRI controls should be done especially in those experiencing local or radicular pains. Dumbbell SS may require extensive bone resection, and giant SS may lead to bony destruction. Posterior instrumentation can be used if instability develops.

\section{- REFERENCES}

1. Abbott R, Feldstein N, Wisoff JH, Epstein FJ: Osteoplastic laminotomy in children. Pediatr Neurosurg 18: 153-156, 1992

2. Celli P, Trillo G, Ferrante L: Spinal extradural schwannoma. J Neurosurg Spine 2: 447-456, 2005

3. Conti P, Pansini G, Mouchaty H, Capuano C, Conti R: Spinal neurinomas: Retrospective analysis and long-term outcome of 179 consecutively operated cases and review of the literature. Surg Neurol 61: 34-43, 2004

4. De Verdelhan O, Haegelen C, Carsin-Nicol B, Riffaud L, Amlashi SF, Brassier G, Carsin M, Morandi X: MR imaging features of spinal schwannomas and meningiomas. I Neuroradiol 32: 42-49, 2005

5. Dorsi MJ, Belzberg AJ: Paraspinal nerve sheath tumors. Neurosurg Clin N Am 15: 217-222, 2004

6. Hagiwara K, Higa T, Miyazato H, Nonaka S: A case of a giant schwannoma on the extremities. J Dermatol 20(11): 700-702, 1993

7. Hori T, Takakura K, Sano K: Spinal neurinomas-clinical analysis of 45 surgical cases. Neurol Med Chir (Tokyo) 24: 471-477, 1984

8. Hsu S, Quattrone M, Ostrom Q, Ryken TC, Sloan AE, Barnholtz-Sloan JS: Incidence patterns for primary malignant spinal cord gliomas: A surveillance, epidemiology, and end results study. J Neurosurg Spine 14:742-747, 2011

9. Javalkar VK, Pigott T, Pal P, Findlay G: Multiple schwannomas. Report of two cases. Eur Spine J 16 Suppl 3: 287-292, 2007

10. Jeon JH, Hwang HS, Jeong JH, Park SH, Moon JG, Kim $\mathrm{CH}$ : Spinal schwannoma; analysis of 40 Cases. J Korean Neurosurg Soc 43(3):135-138, 2008

11. Jinnai $T$, Koyama $T$ : Clinical characteristics of spinal nerve sheath tumors: Analysis of 149 cases. Neurosurgery 56:510515, 2005

12. Kagaya H, Abe E, Sato K, Shimada Y, Kimura A: Giant cauda equina schwannoma. A case report. Spine 25(2):268-272, 2000

13. Kawahara N, Tomita K, Shinya Y, Matsumoto T, Baba H, Fujita T, Murakami H, Kobayashi T: Recapping T-saw laminoplasty for spinal cord tumors. Spine 24:1363-1370, 1999

14. Kim SH, Chin DK, Yoon YS, Jin BH, Cho YE, Kim YS: Spinal instability following for spinal cord tumors: Laminoplasty vs. laminectomy. J Korean Neurosurg Soc 30: S61-S67, 2001

15. Kyoshima K, Horiuchi T, Zenisaka H, Nakazato F: Thoracic dumbbell intra-and extramedullary schwannoma. J Clin Neurosci 12(4): 481-484, 2005

16. Lonstein JE: Post-laminectomy kyphosis. Clin Orthop Relat Res 128: 93-100, 1977

17. Lot G, George B: Cervical neuromas with extradural components: Surgical management in a series of 57 patients. Neurosurgery 41(4): 813-820, 1997

18. Love JG, Dodge HW Jr: Dumbbell (hourglass) neurofibromas affecting the spinal cord. Surg Gynecol Obstet 94:161-172, 1952

19. Mathew P, Todd NV: Intradural conus and cauda equina tumours: A retrospective review of presentation, diagnosis and early outcome. J Neurol Neurosurg Psychiatry 56: 69-74, 1993

20. McCormick PC, Post KD, Stein BM: Intradural extramedullary tumors in adults. Neurosurg Clin N Am 1: 591-608, 1990

21. Park SC, Chung SK, Choe G, Kim HJ: Spinal intraosseous schwannoma: A case report and review. J Korean Neurosurg Soc 46: 403-408, 2009

22. Seppala MT, Haltia MJ, Sankila RJ, Jaaskelainen JE, Heiskanen O: Long-term outcome after removal of spinal schwannoma: A clinicopathological study of 187 cases. J Neurosurg 83: 621-626, 1995

23. Singh DK, Singh N, Rastogi M, Husain M: The transparaspinal approach: A novel technique for one-step removal of dumbbell-shaped spinal tumors. J Craniovertebr Junction Spine 2: 96-98, 2011 